\title{
Patient Perceptions Regarding Multiple Myeloma and Its Treatment: Qualitative Evidence from Interviews with Patients in the United Kingdom, France, and Germany
}

\author{
Jianming He ${ }^{1} \oplus \cdot$ Ashley Duenas ${ }^{2} \cdot$ Hannah Collacott $^{2} \cdot$ Annette Lam $^{1} \cdot$ Katharine S. Gries $^{1} \cdot$ Robin Carson $^{1}$. \\ Dietrich Potthoff ${ }^{3} \cdot$ Nicola Trevor $^{4} \cdot$ Tommi Tervonen $^{2,5}$
}

Accepted: 12 February 2021 / Published online: 9 March 2021

(c) The Author(s) 2021

\begin{abstract}
Background The current standard of care for multiple myeloma requires several regimens of treatment, with patients experiencing high symptom burden and side effects, which negatively impact health-related quality of life (HRQoL). Thus, it is crucial to understand patient perceptions of multiple myeloma and how patients value different treatment options.

Objective The purpose of this study was to conduct an exploratory investigation into concepts that could form attributes that influence treatment choices for patients with multiple myeloma and to identify trade-offs that patients are willing to make between treatment attributes.

Methods In total, 30 patients with newly diagnosed or relapsed/refractory multiple myeloma from the UK, France, and Germany participated in semistructured interviews talking about their disease experience and symptoms, treatment benefits, treatment burden, perceived side effects, and benefit/risk trade-offs in treatment. The interview audio recordings were transcribed and analyzed using content analysis to identify treatment and disease aspects relevant to patients.

Results Symptoms of fatigue and bone pain and treatment side effects of peripheral neuropathy, diarrhea, and constipation were cited by patients as the most disruptive to their HRQoL. Treatment duration was reported most frequently as a major treatment burden, and patients emphasized the importance of increased life expectancy as a treatment benefit. All patients showed good understanding of benefit/risk trade-offs in treatment, and some patients expressed a preference for more convenient modes of treatment administration.

Conclusions Qualitative interviews identified key aspects of multiple myeloma treatment that are most important to patients. These findings will inform a wider patient-preferences study, which could improve treatment choice and HRQoL for patients with multiple myeloma.
\end{abstract}

\section{Key Points for Decision Makers}

This article is part of the topical collection "Formative qualitative evidence for health preference and outcomes research".

Jianming $\mathrm{He}$

jhe2@its.jnj.com

1 Janssen Global Services, LLC, 700 US 202, Raritan, NJ 08869, USA

2 Evidera, London, UK

3 Janssen-Cilag GmbH, Neuss, Germany

4 Janssen-Cilag Ltd, High Wycombe, UK

5 Department of Epidemiology, University Medical Center Groningen, University of Groningen, Groningen, The Netherlands
This research identified the most relevant concepts related to multiple myeloma and its treatment, including symptom burden, treatment duration, and side effects, that patients may consider when choosing between different treatment options.

These findings will be used to inform a discrete-choice experiment that will yield quantitative data regarding treatment preferences in patients with multiple myeloma. 


\section{Introduction}

Multiple myeloma (MM) is the second most common hematological cancer and is characterized by high levels of mortality [1-4]. Despite recent treatment advances, MM remains incurable [1-3, 5-8] and becomes harder to treat over time $[2,3,8]$. Development of new treatment options, particularly those that can be tolerated over the long term, is an ongoing priority. The current standard of care requires multiple treatment regimens; with chronic treatment, patients experience cumulative toxicities and high symptom burden, including bone pain, fatigue, and peripheral neuropathy $[1,2,4,5,7,9]$. Little is known about how patients weigh the benefits and risks of a treatment and which aspects are important to them [10]. Longterm struggles in coping with symptoms, and difficulties in managing treatment, lead to a substantial negative impact on patients' health-related quality of life (HRQoL) [2, 4, $8,9]$. Studies in European populations report significantly lower scores on several aspects of HRQoL, including increased pain and fatigue and reduced physical and social functioning, compared with an age- and sex-matched population [11, 12]. In addition, patients with $\mathrm{MM}$ also report significant levels of anxiety, depression, and psychosocial stress [13].

Given the difficulties of MM management and treatment, it is crucial to understand how patients value different treatment options. Recent MM research has started to focus on patient HRQoL and attitudes toward treatment regimens $[4,5,10]$. However, current information about the preferences of patients with MM is limited. To date, little attention has been given to the disease experiences or treatment preferences of patients with MM or how those preferences are shaped [3]. There is a paucity of research on treatment preferences of patients with newly diagnosed MM (NDMM) [3, 14].

In recent years, health regulatory authorities have stressed the importance of incorporating patients' perspectives in the development of new treatments $[15,16]$. Discrete-choice experiments (DCEs) are one type of preference-elicitation method that can be used to assess the relative value of individual features that may differ between treatment options. DCEs ask participants to choose between multiple-choice alternatives described in terms of attributes that vary between the choice tasks. DCEs are used in health economics to assess the treatment attributes that matter to patients and how much of one attribute respondents are willing to give up to gain more (or less) of another attribute. Policy makers have shown increased interest in using DCEs for treatment decision-making $[17,18]$.

The purpose of this study was to conduct an exploratory investigation into concepts that could form attributes that influence treatment choices for patients with MM. In addition, we examined whether patients are willing to trade-off benefits and risks for more convenient modes of administration. This study comprises part one of a two-part study to elicit patients' benefit/risk trade-offs on key efficacy and safety outcomes of MM treatments. The first part included a literature review followed by a qualitative interview, designed to inform instrument development, and will end in the second part, the DCE, which is currently underway. Results from the qualitative interviews are presented here and are crucial to establishing a quantitative methodology for measurement of the treatment preferences of patients with MM.

\section{Methods}

\subsection{Literature Review}

A targeted literature review (electronic supplementary material [ESM]-1) was conducted to develop an extensive list of attributes relevant to the benefits and risks of MM treatment to inform the conceptual development of an interview guide and vignettes (Fig. 1). Attributes within each of the following categories were considered for inclusion in the qualitative interviews: clinical benefit, HRQoL, adverse events (AEs), and convenience.

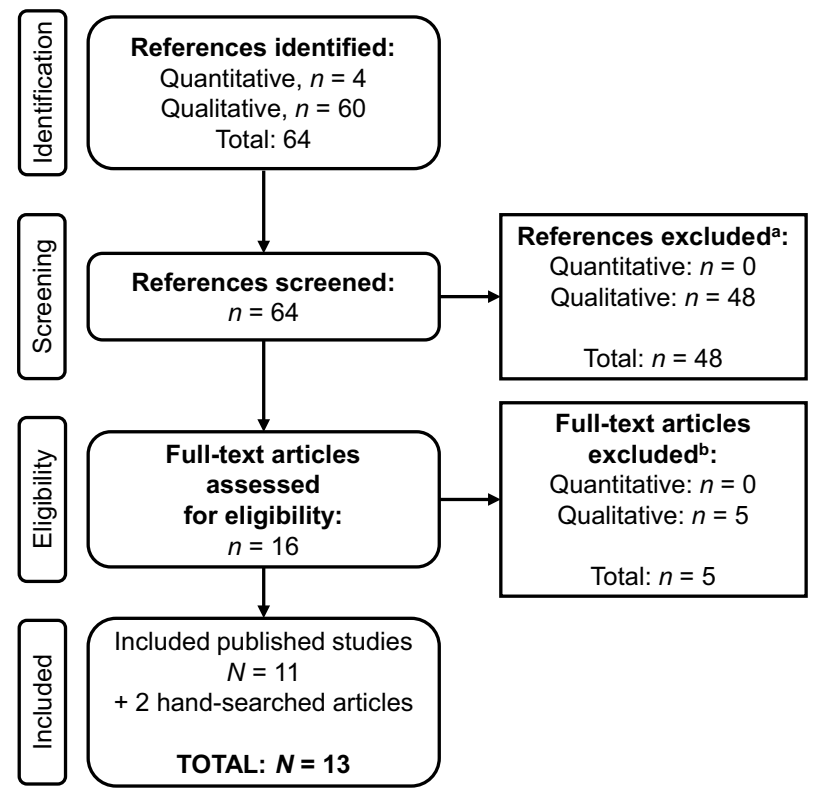

Fig. 1 Literature review PRISMA (Preferred Reporting Items for Systematic Reviews and Meta-Analyses) flowchart. ${ }^{a}$ References were excluded for the following reasons: study population not relevant $(n=12)$, study design not relevant $(n=33)$, and outcome not relevant $(n=3)$. ${ }^{\mathrm{b}}$ Studies were excluded if study design was not relevant 


\subsection{Study Population}

Eligible patients were aged $\geq 18$ years, resided in the UK, France, or Germany, and had a physician-confirmed MM diagnosis. Participants included patients with transplantineligible (TIE) NDMM, transplant-eligible (TE) NDMM, or relapsed/refractory MM (RRMM). Those with primary amyloid light chain amyloidosis, monoclonal gammopathy of undetermined significance, or smoldering MM were excluded from the study.

As the study was qualitative, the sample size estimation was based on a purposive sampling approach. We sought to gain rich qualitative data in a diverse sample of patients with NDMM and RRMM from three countries, requiring a smaller sample size than found in quantitative research. The sample size of each analysis group $(n=10)$ was fixed in advance to ensure saturation of concepts within analysis groups while considering a feasible sample size in the target population $[19,20]$. We identified a priori that we would review the first five interviews in the first round of analysis, allowing us to include additional probes or modify the guide as needed; the second round of interviews allowed for an additional 20 interviews to reach saturation. This method aligned with recommendations for qualitative research [19].

\subsection{Recruitment Procedures}

To ensure a diverse sample, patients were recruited through varied sources, including physician referrals, patient panels, patient associations and advocacy groups, and social media. Standardized study invitations informed potentially eligible patients about the purpose of the research, the length of the interviews (approximately $60 \mathrm{~min}$ ), and their remuneration for participation. Once potential participants expressed interest in the study, participant eligibility was verified via telephone using a screening script. The screening script and emails to patients were worded generically to prevent biases in responsiveness. During screening, recruiters explained the rationale of the study. Relationships between some participants and recruiters existed in cases where they were members of recruiter patient panels. Beyond this, no relationships were established prior to study commencement (i.e., invitation, screening, scheduling). To verify the MM diagnosis, all participants who passed prescreening verbally consented to provide a proof of diagnosis form signed by a healthcare provider. This form included questions on classification of MM, eligibility for transplant, current treatment and treatment history, and Eastern Cooperative Oncology Group (ECOG) performance status. Eligible participants were scheduled for a one-time 60-minute telephone interview.

Participants provided informed consent prior to taking part in the study. The study protocol and the informed consent forms received ethical approval from a central institutional review board. The original approval was received on 5 March 2019 (approval number 18200).

\subsection{Interviews}

Qualitative interviews were conducted with 30 patients in two rounds via telephone using a semistructured interview guide developed from a targeted literature review and a conceptual framework [2]. Five patients were interviewed in the first round; the interview guide was then revised based on the feedback obtained from these patients. This revised interview guide was used for the second round of interviews. The first half of the interview guide included open questions related to the patients' experiences with MM to identify any new themes/concepts, whereas the second half of the interview guide included semistructured questions related to treatment experiences based on the literature review and questions related to risk/benefits of treatments from the vignettes. Interviews were audio recorded. Participants reviewed two different hypothetical vignettes to discuss how they would choose between different treatment options. Additional information on interviews and vignettes presented to patients are included in ESM-2.

\subsection{Data Analysis}

Audio recordings from interviews were transcribed and reviewed for quality assurance. Interviews in French and German were translated into English before analysis.

Concepts were coded using the ATLAS.ti 8.0 [21]. Two Evidera researchers, AD and $\mathrm{HC}$, used content analysis methods to identify passages of text in the transcripts for themes at the explicit or surface-level meaning [22]. Quotes that illustrated the words and phrases used by the study participants when explaining these concepts were coded using a coding dictionary and grouped into key themes. The coding dictionary was developed based both on the interview guide and on a conceptual framework developed by Baz et al. [2]. It was updated iteratively to reflect themes emerging throughout the analysis. Consistency of concepts elicited were documented through a "saturation grid" to identify the (non-)emergence of new themes. The point of saturation was assessed with support from the data that no substantially new information was emerging with additional interviews [2, $19,23]$ (the symptoms saturation grid is provided in ESM3). Descriptive analyses were conducted in R 3.6.1 software to explore the frequency of concepts related to symptoms, benefits, and side effects as well as patterns between subgroups. Demographic and clinical data were analyzed with descriptive statistics using StataMP ${ }^{\circledR}$ version 15.0 or higher. Results were reported for all participants and for the participant subgroups (TE NDMM, TIE NDMM, RRMM). 


\subsection{Qualitative Study Design and Reporting Guidelines}

Study design and data reporting were conducted in line with published guidelines for reporting formative qualitative research [20, 24, 25]. The data elements captured from the studies followed the STROBE (STrengthening the Reporting of OBservational studies in Epidemiology) guidelines for cohort and cross-sectional studies, including study type, study characteristics, population/subpopulations of interest, patient characteristics, and categories of outcomes reported.

\section{Results}

\subsection{Participants}

In total, 30 patients from the UK $(40 \% ; n=12)$, France $(26.7 \% ; n=8)$, and Germany $(33.3 \% ; n=10)$ were interviewed between June and September 2019. Patient characteristics are shown in Table 1 . Of the study participants, $60 \%(n=18)$ had NDMM and 40\% $(n=12)$ had RRMM. Of the patients with NDMM, 66.7\% $(n=12)$ were TE and $33.3 \%(n=6)$ were TIE. The mean age of the patients was
60.3 years; $50 \%(n=15)$ were male, and $80 \%(n=24)$ had an ECOG performance status of 0 or 1 , indicating minimal physical impairment.

\subsection{Symptoms}

In the study interviews, patients discussed a wide range of symptoms (Table 2). Representative quotes from patient interviews are shown in ESM-4. The MM symptoms most often discussed were bone pain $(n=27 ; 90 \%)$, fatigue ( $n=26 ; 87 \%$ ), and "tingling hands or feet/restless leg" (peripheral neuropathy; $n=9 ; 30 \%$ ). Common themes in responses were observed among all patients, although there were some notable differences between NDMM and RRMM subgroups. Bone pain and fatigue were the two most commonly discussed symptoms. On the symptom of fatigue, one patient with NDMM stressed,

"Fatigue. I was very, very tired. Sometimes, I sit in my armchair for 5-10 min and have a short nap, and then I feel better. But I am always very tired, all day long. I can no longer work around the house as I used to, do what I used to do - it's 'fatigue,' physical, and I can no longer do much.” (TIE NDMM, France)

Table 1 Participant characteristics

\begin{tabular}{|c|c|c|c|c|}
\hline Characteristics & Total $(N=30)$ & NDMM-TE $(n=12)$ & NDMM-TIE $(n=6)$ & $\operatorname{RRMM}(n=12)$ \\
\hline Male & $15(50.0)$ & $5(41.7)$ & $4(66.7)$ & $6(50.0)$ \\
\hline Age, years & $60.3 \pm 10.7$ & $56.8 \pm 12.6$ & $67.3 \pm 6.8$ & $60.4 \pm 9.1$ \\
\hline Employed full or part time & $8(26.7)$ & $5(41.7)$ & $1(16.7)$ & $2(16.7)$ \\
\hline \multicolumn{5}{|l|}{ Country } \\
\hline UK & $12(40.0)$ & $6(50.0)$ & $1(16.7)$ & $5(41.7)$ \\
\hline France & $8(26.7)$ & $3(25.0)$ & $2(33.3)$ & $3(25.0)$ \\
\hline Germany & $10(33.3)$ & $3(25.0)$ & $3(50.0)$ & $4(33.3)$ \\
\hline First line of therapy & $15(50.0)$ & $9(75.0)$ & $6(100.0)$ & 0 \\
\hline \multicolumn{5}{|l|}{ ECOG performance status } \\
\hline 0 & $10(33.3)$ & $4(33.3)$ & $1(16.7)$ & $5(41.7)$ \\
\hline 1 & $14(46.7)$ & $7(58.3)$ & $4(66.7)$ & $3(25.0)$ \\
\hline$\geq 2$ & $6(20.0)$ & $1(8.3)$ & $1(16.7)$ & $4(33.3)$ \\
\hline \multicolumn{5}{|l|}{ Current medications } \\
\hline PI & $20(66.7)$ & $7(58.3)$ & $5(83.3)$ & $8(66.7)$ \\
\hline IMiD & $17(56.7)$ & $7(58.3)$ & $2(33.3)$ & $8(66.7)$ \\
\hline Chemotherapy $^{\mathrm{a}}$ & $9(30.0)$ & $4(33.3)$ & $4(66.7)$ & $1(8.3)$ \\
\hline Steroids & $21(70.0)$ & $7(58.3)$ & $6(100.0)$ & $8(66.7)$ \\
\hline CD-38 inhibitor & $5(16.7)$ & $2(16.7)$ & 0 & $3(25.0)$ \\
\hline Other $^{\mathrm{b}}$ & $4(13.3)$ & $1(8.3)$ & 0 & $3(25 \%)$ \\
\hline
\end{tabular}

Data are presented as mean \pm standard deviation or $n(\%)$ unless otherwise indicated

$C D$ cluster of differentiation, ECOG Eastern Cooperative Oncology Group, IMiD immunomodulatory drug, NDMM newly diagnosed multiple myeloma, $P I$ proteasome inhibitor, RRMM relapsed/refractory multiple myeloma, TE transplant eligible, TIE transplant ineligible

${ }^{a}$ Bendamustine, cisplatin, cyclophosphamide, doxorubicin, etoposide, melphalan

${ }^{\mathrm{b}}$ Elotuzumab, panobinostat, no treatment 
Table 2 Most commonly discussed symptoms in the overall population and by disease stage

\begin{tabular}{llll}
\hline Symptom & Total $(N=30)$ & NDMM $(n=18)$ & RRMM $(n=12)$ \\
\hline Bone pain & $27(90.0)$ & $17(94.0)$ & $10(83.0)$ \\
Fatigue/tiredness & $26(87.0)$ & $16(89.0)$ & $10(83.0)$ \\
$\begin{array}{l}\text { Tingling hands or } \\
\text { feet or restless }\end{array}$ & $9(30.0)$ & $5(28.0)$ & $4(33.0)$ \\
$\quad$ leg & & & \\
Infection & $8(27.0)$ & $4(22.0)$ & $4(33.0)$ \\
Sleepiness & $4(13.0)$ & $2(11.0)$ & $2(17.0)$ \\
Constipation & $4(13.0)$ & $3(17.0)$ & $1(8.0)$ \\
Muscle cramps & $3(10.0)$ & $2(11.0)$ & $1(8.0)$ \\
Headache & $3(10.0)$ & 0 & $3(25.0)$ \\
Insomnia & $3(10.0)$ & $3(17.0)$ & 0 \\
\hline
\end{tabular}

Data are presented as $n(\%)$. Symptoms discussed in more than one interview; includes both spontaneous and prompted mentions; one or more "other" symptoms (including anemia, bleeding, circulatory issues, cognitive issues, depression, diarrhea, dizziness, fainting, hair loss, heartburn, joint pain, lack of motivation, nausea, night sweats, nosebleeds, one-sided weakness, osteoporosis, other bone [e.g., dissolution, fracture], popliteal cyst, shortness of breath, sweating, tinnitus, weakness, uncertainty on feet, vision problems, vomiting, and weight loss) were mentioned in 15 (50.0\%) interviews

$N D M M$ newly diagnosed multiple myeloma, RRMM relapsed/refractory multiple myeloma

Both NDMM and RRMM groups discussed bone pain and fatigue, but greater proportions of patients with NDMM discussed these compared with those with RRMM. Pain was also described differently among patients. Some focused on the location (e.g., "back pain"). The severity of pain mentioned ranged from "manageable" to "severe" and "unbearable." One patient with NDMM commented,

"I literally was screaming in 'pain,'; I was on my own in the house at the time and my family were really worried about me, but I was literally, 'I couldn't walk, I was almost paralyzed,' this came on so suddenly." (TE NDMM, UK)

The symptom of headache was only mentioned by a small number of patients with RRMM $(n=3 ; 25 \%)$. In contrast, insomnia and dizziness were only discussed by patients with $\operatorname{NDMM}(n=3 ; 17 \%)$. Notably, there was overlap between symptoms of MM and side effects from treatment(s). Some were unsure whether peripheral neuropathy, fatigue, or infections were due to MM or its treatment.

\subsection{Impacts}

Patients most often discussed the impact of MM on daily life ( $n=23 ; 77 \%)$, a broad concept encompassing a range of day-to-day activities, including the ability to perform household tasks, such as cooking, cleaning, and gardening.
Table 3 Most commonly discussed impacts in the overall population and by disease stage

\begin{tabular}{llll}
\hline Impact & Total $(N=30)$ & NDMM $(n=18)$ & RRMM $(n=12)$ \\
\hline Daily life & $23(77.0)$ & $14(78.0)$ & $9(75.0)$ \\
Physical activity & $22(73.0)$ & $16(89.0)$ & $6(50.0)$ \\
Social life & $19(63.0)$ & $10(56.0)$ & $9(75.0)$ \\
$\begin{array}{l}\text { Emotional } \\
\text { general }\end{array}$ & $15(50.0)$ & $11(61.0)$ & $4(33.0)$ \\
Work & $10(33.0)$ & $7(39.0)$ & $3(25.0)$ \\
Emotional anxi- & $8(27.0)$ & $5(28.0)$ & $3(25.0)$ \\
$\quad$ ety & & & $1(8.0)$ \\
$\begin{array}{l}\text { Insomnia/sleep } \\
\text { Family life }\end{array}$ & $6(20.0)$ & $5(28.0)$ & $2(17.0)$ \\
Emotional & $6(20.0)$ & $4(22.0)$ & $2(17.0)$ \\
$\quad$ depression & $3(10.0)$ & $1(6.0)$ & \\
\hline
\end{tabular}

Data are presented as $n(\%)$. Impacts discussed in more than one interview; includes both spontaneous and prompted mentions; one or more "other" impacts (including cognitive issues, future planning, impact on studies, lack of motivation, mental capacity/time management, pain/medication, and sensitivity to foods) were mentioned in ten $(33.0 \%)$ interviews

NDMM newly diagnosed multiple myeloma, RRMM relapsed/refractory multiple myeloma

Impacts on physical activity and social life were also frequently mentioned (Table 3). Responses were generally consistent between patients with NDMM and those with RRMM, although a greater proportion of patients with NDMM $(n=16 ; 89 \%)$ discussed the impact on physical activity compared with patients with RRMM $(n=6 ; 50 \%)$. In addition, a greater proportion of patients with RRMM $(n=9$; $75 \%$ ) discussed the impact of MM on social life compared with patients with NDMM $(n=10 ; 56 \%)$. To illustrate, one patient commented,

"In the early days when I was sort of new out of treatment or on treatment it 'prevented me going out as much' because I'd be tired or I wouldn't feel well or the 'taste in your mouth means you don't want to sort of eat or drink' so that ... you 'stop looking forward to going out' so much because you knew you wouldn't enjoy it." (RRMM, UK)

Another patient commented,

"There have been cases 'where I have had to cancel a meeting with someone. Simply because I didn't want to force myself to go.' You bring sort of a bad mood with you. You know? 'Because you yourself aren't feeling so well. Not in such a good mood.' And then you say, 'No, don't get mad, but today I really don't feel like it.' My good friends know this and they accept it. But otherwise, it's a little bit difficult." (RRMM, Germany) 


\subsection{Treatment Benefits}

When asked to discuss which improvements they would value most in a given treatment, the benefit most often discussed was increased life expectancy $(n=26 ; 87 \%$; Table 4$)$. Many patients referred to their desire to extend their life in order to have more time with family (predominantly children and grandchildren). Patients also noted remission/response and reduced fatigue (each $n=24 ; 80 \%$ ) as valuable treatment benefits. Among patients with NDMM, reduced fatigue was the most often discussed treatment benefit ( $n=16 ; 89 \%)$, whereas among patients with RRMM, increased life expectancy was discussed most often $(n=11 ; 92 \%)$. Notably, more patients with NDMM $(n=15 ; 83 \%)$ discussed independence as an important treatment benefit compared with patients with RRMM ( $n=6 ; 50 \%)$. Overall, consistent themes were reported by patients relating to improved HRQoL. Many patients stressed treatment benefits such as being able to carry out "normal activities." When asked about treatment benefits, one patient commented,

"I don't know, that's really hard to say because like day to day I'd say, 'reducing the fatigue,' but actually it's more important to 'suppress the disease as soon as possible.' So, I'd probably say that I probably think it's probably 'better to deal with the fatigue and suppress the disease quicker."” (TE NDMM, UK)

\subsection{Treatment Side Effects}

Overall, the side effects most often discussed were peripheral neuropathy $(n=27 ; 90 \%)$, diarrhea/constipation $(n=25 ; 83 \%)$, and cognitive impairment $(n=25 ; 83 \%$; Table 5). Patients with RRMM and those with NDMM differed slightly in their discussions of side effects, with more patients with RRMM reporting peripheral neuropathy $(n=11 ; 92 \%)$ and swelling of hands and feet $(n=11 ; 92 \%)$. Most patients with NDMM reported cognitive impairment $(n=17 ; 94 \%)$, followed by peripheral neuropathy $(n=16$; $89 \%$ ).

As noted in Sect. 3.2, there was overlap between the symptoms of MM and side effects from treatment. Notable overlap was observed in many symptoms associated with peripheral neuropathy (sensory, motor, autonomic). To illustrate, one patient commented,

"For the past four months, I've been undergoing chemotherapy. That means that 'many symptoms have been caused by side effects from medications.' They cannot be solely traced to the illness, or it's difficult to distinguish them. During certain phases, depending on what medications I'm taking, I have an increase in 'muscle cramps.' I've got 'polyneuropathy with a variety of symptoms.'” (TE NDMM, Germany)

Patients referenced the frequency or duration of symptoms when thinking about the acceptability of side effects. Some patients reported a willingness to accept short-term severe side effects from a treatment that would extend their lifespan. The most frequently mentioned side effect was
Table 4 Most commonly discussed treatment benefits in the overall population and by disease stage

\begin{tabular}{llll}
\hline Treatment benefit & Total $(N=30)$ & NDMM $(n=18)$ & RRMM $(n=12)$ \\
\hline Increased life expectancy & $26(87.0)$ & $15(83.0)$ & $11(92.0)$ \\
Remission/response & $24(80.0)$ & $14(78.0)$ & $10(83.0)$ \\
Reduced fatigue & $24(80.0)$ & $16(89.0)$ & $8(67.0)$ \\
Reduced worry & $22(73.0)$ & $13(72.0)$ & $9(75.0)$ \\
Independence & $21(70.0)$ & $15(83.0)$ & $6(50.0)$ \\
Increased time to recurrence & $21(70.0)$ & $13(72.0)$ & $8(67.0)$ \\
Reduced bone pain & $21(70.0)$ & $14(78.0)$ & $7(58.0)$ \\
Time to response & $20(67.0)$ & $12(67.0)$ & $8(67.0)$ \\
Improved social life & $18(60.0)$ & $13(72.0)$ & $5(42.0)$ \\
Planning for the future & $18(60.0)$ & $11(61.0)$ & $3(58.0)$ \\
Improved ability to work & $12(40.0)$ & $9(50.0)$ & $5(42.0)$ \\
Health-related quality of life & $10(33.0)$ & $5(28.0)$ & $4(33.0)$ \\
Reduced dependence (self-care) & $8(27.0)$ & $4(22.0)$ & \\
\hline
\end{tabular}

Data are presented as $n(\%)$. Treatment benefits discussed in more than one interview; includes both spontaneous and prompted mentions; one or more "other" benefits (including being able to return to "normal" life/daily activities, being drug free, cure, improved appetite, improved blood results, improved sleep, less frequent treatment, less time in hospital, no doctors' appointments, reduced likelihood of bone fractures, reduced symptoms [general], and reduced weakness) were mentioned in $19(63.0 \%)$ interviews

$N D M M$ newly diagnosed multiple myeloma, $R R M M$ relapsed/refractory multiple myeloma 
Table 5 Most commonly discussed treatment side effects in the overall population and by disease stage

\begin{tabular}{lccc}
\hline Treatment side effect & Total $(N=30)$ & NDMM $(n=18)$ & RRMM $(n=12)$ \\
\hline Peripheral neuropathy & $27(90.0)$ & $16(89.0)$ & $11(92.0)$ \\
Diarrhea/constipation & $25(83.0)$ & $15(83.0)$ & $10(83.0)$ \\
Cognitive impairment & $25(83.0)$ & $17(94.0)$ & $8(67.0)$ \\
Nausea/vomiting & $23(77.0)$ & $15(83.0)$ & $8(67.0)$ \\
Swelling of hands and feet & $23(77.0)$ & $12(67.0)$ & $11(92.0)$ \\
Risk of infection & $23(77.0)$ & $15(83.0)$ & $8(67.0)$ \\
Hematologic & $18(60.0)$ & $12(67.0)$ & $6(50.0)$ \\
Fatigue & $17(57.0)$ & $12(67.0)$ & $5(42.0)$ \\
Kidney infection & $3(10.0)$ & $2(11.0)$ & $1(8.0)$ \\
Fevers/infections & $2(7.0)$ & $1(6.0)$ & $1(8.0)$
\end{tabular}

Data are presented as $n(\%)$. Treatment side effects discussed in more than one interview; includes both spontaneous and prompted mentions; one or more "other" side effects (including amyloid lumps, back pain, breathlessness, cramps, depression, diabetic ketoacidosis, dizziness, dry skin, deep venous thrombosis, fatigue, fractures, hair loss, headache, hearing loss, heart problems, heartburn, insomnia, leg pain, menopause, mouth ulcers, mucosal dryness, night sweats, organ damage, rashes, reduced strength, secondary cancers, sense of taste, sweats, tinnitus, vertigo, vision problems, weight gain, weight loss) were mentioned in $28(93.0 \%)$ interviews

$N D M M$ newly diagnosed multiple myeloma, $R R M M$ relapsed/refractory multiple myeloma cognitive impairment for patients with NDMM $(n=17$; 94\%) and peripheral neuropathy for patients with RRMM $(n=11 ; 92 \%)$. Notably, patient understanding of the phrase "toxicity" was mixed, and participants' responses to probing on their understanding of toxicity versus side effects often did not elicit a clear definition.

\subsection{Treatment Burden}

Regardless of disease stage, the treatment burden most often discussed was the duration of individual treatment administrations, including the length of time required to travel to receive treatments and the length of time required for intravenous infusions. In total, $83 \%(n=15)$ of patients with NDMM and 75\% ( $n=9)$ of those with RRMM mentioned treatment duration during their interviews (Table 6). Patients also emphasized the long distances between their homes and the local hospital, as well as the time required to travel to and from the hospital for treatment. This burden led many patients to prefer home-administered therapies to those administered in a hospital.

"It's a bit burdensome in the long term as it's supposed to be twice a week, and 'it requires going to the hospital twice a week,' so it feels like we're living with HCPs [healthcare professionals] on a daily basis. 'It's a bit burdensome." (RRMM, France)

Patients with RRMM and those with NDMM did not greatly differ in their discussion of treatment burdens. The most notable differences were found among patients
Table 6 Most commonly discussed treatment burdens in the overall population and by disease stage

\begin{tabular}{|c|c|c|c|}
\hline Treatment burden & Total $(N=30)$ & $\operatorname{NDMM}(n=18)$ & $\operatorname{RRMM}(n=12)$ \\
\hline $\begin{array}{l}\text { Treatment dura- } \\
\text { tion }\end{array}$ & $24(80.0)$ & $15(83.0)$ & $9(75.0)$ \\
\hline Location/travel & $22(73.0)$ & $14(78.0)$ & $8(67.0)$ \\
\hline $\begin{array}{l}\text { Intravenous } \\
\text { injection }\end{array}$ & $13(43.0)$ & $8(44.0)$ & $5(42.0)$ \\
\hline $\begin{array}{l}\text { Subcutaneous } \\
\text { injection }\end{array}$ & $6(20.0)$ & $6(33.0)$ & 0 \\
\hline Other side effect & $6(20.0)$ & $4(22.0)$ & $2(17.0)$ \\
\hline Monitoring & $3(10.0)$ & $3(17.0)$ & 0 \\
\hline $\begin{array}{l}\text { Oral administra- } \\
\text { tion }\end{array}$ & $2(7.0)$ & $1(6.0)$ & $1(8.0)$ \\
\hline
\end{tabular}

Data are presented as $n(\%)$. Treatment burdens mentioned in one or more interview; includes both spontaneous and prompted mentions; "other" treatment burdens (including dependency on medication and organization required to take medications) were mentioned in four (13.0\%) interviews

NDMM newly diagnosed multiple myeloma, RRMM relapsed/refractory multiple myeloma

with NDMM, who reported subcutaneous injection ( $n=6$; $33 \%)$ and monitoring $(n=3 ; 17 \%)$ as burdens, whereas no patients with RRMM reported these burdens. Patients' opinions on where to have the treatment administered were divided. Some felt that hospital-based treatments were associated with a higher risk of infections, and others reported feeling more comfortable receiving treatment in a hospital setting in case anything should go wrong. 


\subsection{Vignettes}

Overall, patients demonstrated a good level of understanding of the information provided in the vignettes. Patients understood what treatment benefits, such as increased life expectancy and remission, would mean. All understood the risk data shown to them and seemed comfortable with interpreting risk as a percentage. However, patients reported that further details regarding side effects would have been beneficial to better inform their decision regarding treatment. Patients also mentioned the need to clarify details regarding treatment administration and information on the schedule associated with the appointments. Some patients expressed uncertainty about the appointment time for subcutaneous injections, suggesting this was longer than they might expect. Overall feedback was that patients would prefer injections over infusions (vignette B), although, when probed, they reported that they would be willing to accept whichever treatment was available at the time.

Patients expressed a willingness to accept severe or mild to moderate treatment risks at different thresholds. Not all patients provided comments on threshold levels because of limited time in the interviews and the fact that the question was only probed after the second round of interviews. In total, $70 \%(n=21)$ of patients commented on how willing they would be to accept a treatment with potentially severe AEs. Only four patients (three with NDMM, one with RRMM) were willing to accept a treatment with a $>40 \%$ risk of severe AEs. Eight patients were willing to accept a treatment with a $30-40 \%$ risk of severe AEs, and nine were willing to accept a treatment with a $<30 \%$ risk of severe AEs. A total of $60 \%(n=18)$ of patients commented on how willing they would be to accept a treatment with mild to moderate AEs. Of these, only five patients (four with NDMM, one with RRMM) were willing to accept a treatment with a $>75 \%$ risk of mild to moderate AEs. Eight patients (five with NDMM, three with RRMM) were willing to accept a treatment with a $75 \%$ risk of mild to moderate AEs, whereas another five (one with NDMM, four with RRMM) were only willing to accept a treatment with $a<75 \%$ risk of mild to moderate AEs.

\section{Discussion}

With increasing treatment options available and various complexities involved, it is crucial to understand patient preferences in the decision-making process to provide the best possible treatment for patients with MM. This study focused on the experiences of a diverse group of patients with NDMM and RRMM from the UK, France, and Germany, providing insights into the symptoms and impacts of MM. In addition, this study highlighted the treatment benefits, side effects, and treatment burdens that matter most to patients.

Patients in this study discussed a variety of symptoms and treatment side effects that impacted their daily lives, emotional health, physical activities, and social lives and diminished their HRQoL (Fig. 1 in ESM-1; ESM-3). The results presented here add to the existing literature on patient experiences with $\mathrm{MM}$ and preferences for MM treatment. Additionally, new insights about patients' concerns related to "chemotherapy brain" were identified that were more prominent in patients with RRMM. Other newly identified themes included burden of traveling for treatment and overlap between AEs and symptoms such as peripheral neuropathy, where patients were unsure whether they were experiencing disease symptoms or treatment-related AEs. In agreement with previous studies on treatment preferences in patients with MM [3, 10], participants prioritized increased life expectancy as a treatment benefit of utmost importance. As in previous studies, patients reported high symptom burden, including symptoms associated with HRQoL $[1,2,4,5$, $7,9]$. In agreement with another qualitative study on treatment preferences in MM [3], symptoms such as fatigue and bone pain, and side effects such as gastrointestinal symptoms and peripheral neuropathy, were the most frequently discussed by patients as significantly disruptive to HRQoL. In both the present study and in Parsons et al. [3], patients stressed the value of improved quality of life overall and being able to carry out normal daily activities as a result of treatment. In contrast to Parsons et al. [3], patients with MM in our study did not discuss the financial impact of treatment as a major burden. This was likely because of the prevalence of national medical coverage in the countries in which our patient interviews were conducted.

Existing research on patient preferences in MM has focused on RRMM populations [3, 10]; our study included patients with NDMM as well as those with RRMM. By including patients from different disease settings, we were able to identify key similarities and differences between patients with NDMM and those with RRMM in our analysis. For symptoms, all patient subgroups reported fatigue, bone pain, and peripheral neuropathy; however, patients with NDMM frequently mentioned bone pain and fatigue, whereas patients with RRMM most commonly discussed peripheral neuropathy and infections, which can be associated with the safety profiles of certain MM regimens. Although side effects such as peripheral neuropathy and gastrointestinal issues were a common concern in all subgroups, cognitive burden appeared to be more of a concern for newly diagnosed patients. When considering the length of duration and treatment procedures, patients with NDMM commented that they preferred subcutaneous injection over infusion to a greater extent than patients with RRMM. In addition, increased life expectancy was discussed as an important 
treatment benefit for all participants. Newly diagnosed patients tended to mention reduced fatigue and independence more often than patients with RRMM. A recent analysis of different stages of MM reported that HRQoL seemed to improve over time in MM, with patients with RRMM showing better global health status and better emotional, physical, and role functioning than those newly diagnosed, which suggests patients may learn to cope with the disease over time [6]. The authors suggested one possible explanation might be a shift in patients' HRQoL expectations as the disease progresses. Along these lines, our results indicate that patients with NDMM may be more burdened by certain symptoms, such as pain and fatigue, and side effects such as cognitive burden, than those with RRMM. In addition, patients with NDMM may value convenience of administration to a greater extent than those with RRMM when choosing MM treatments. Notably, some regional differences in views about treatment burden and benefit were observed, although conclusions about such differences are limited by small sample sizes.

In a recent study assessing patient preferences for RRMM therapies, patients facing trade-offs were willing to accept a higher AE rate or shorter progression-free time to get an oral treatment over other modes of administration [26]. Similarly, our qualitative analysis offers valuable patient feedback on the maximum acceptable risk levels for mild to moderate and severe side effects of both NDMM and RRMM treatments. Thus, this qualitative study is a crucial step toward a larger endeavor to establish quantitative methods to elicit patients' benefit/risk trade-offs on key efficacy and safety outcomes of MM treatments. Results from this qualitative part of the study were used to identify attributes for use in the quantitative part (a DCE), which is currently in progress. Concepts raised as particularly important or bothersome to patients were considered for inclusion as attributes alongside other criteria. An important lesson learned from this study was that severity of fatigue and pain, in addition to frequency, significantly impacts patient HRQoL. This finding may provide insights for future MM patient-reported outcome instrument enhancement.

In addition to informing attribute selection, qualitative insights from the interviews were used to inform attribute descriptions and levels to ensure the language used in future work is most relevant to patients. Results from this study showed patients demonstrated no difficulty in understanding the vignettes or the risk-threshold levels presented; however, when probed on terms such as "toxicity" and "depth of response," patients' level of understanding of the terminology varied. The importance of using plain language in patient communication and education is wellestablished [27, 28], and future studies should utilize plain language terms when developing attribute levels for ease of understanding.
As this study was exploratory in nature, it had certain limitations. A qualitative study design was chosen to serve the study's primary purpose of understanding and confirming the patient experience of MM. In addition, the qualitative design functions as an inductive approach to inform later aspects of the research, which will utilize quantitative methods. Sample sizes were small but were purposive and limited to specific subgroups of patients with MM. Patients were recruited through varied sources to ensure a diverse sample, although patients recruited through these methods may differ from the general MM population.

\section{Conclusions}

The qualitative interviews in this study identified aspects of $\mathrm{MM}$ and its treatment that are most important to patients in the UK, France, and Germany. Patients stressed the symptoms of fatigue and bone pain and the treatment side effects of peripheral neuropathy, diarrhea, and constipation as the most disruptive to HRQoL. In addition, treatment duration was cited most frequently as a major treatment burden. Finally, patients emphasized the importance of the treatment benefit of increased life expectancy in their interviews. These findings will inform future research to identify and characterize patient treatment preferences, which could potentially impact treatment choice and improve HRQoL for patients with NDMM and RRMM.

Supplementary Information The online version contains supplementary material available at https://doi.org/10.1007/s40271-021-00501-7.

Acknowledgements Medical writing support was provided by Corey Eagan, MPH, of Eloquent Scientific Solutions and was funded by Janssen Global Services, LLC. Sarah Cote, Xenia Gatopoulou, Caroline Mckay, Marta Pisini, Mary Slavcev, and Ralph Riley (Janssen) reviewed the study protocol. French patient interviews were moderated by Sonia Halhol (Evidera), and German interviews were moderated by Brigitte Schrödel (Schrödel Market Research). Gin-Nie Chua, Dina Filipenko, and Charlie Smith (Evidera) supported data analysis for this study. Sumitra Sri Bhashyam (formerly Evidera) provided valuable support in study conception and design.

\section{Declarations}

Funding This study was funded by Janssen Global Services, LLC.

Conflicts of interest Jianming He, Annette Lam, Katherine Gries, Robin Carson, and Nicola Trevor are employees of Janssen and own company stocks. Ashley Duenas, Hannah Collacott, and Tommi Tervonen are employees of Evidera, which received funding from Janssen for work related to this study. Dietrich Potthoff is an employee of Janssen.

Availability of data and material The datasets generated and/or analyzed during the current study are not publicly available because no consent was sought from participants to allow sharing of data with third parties. 
Ethics approval The study protocol and the informed consent forms received ethical approval from a central institutional review board (Ethical and Independent Review Services, Independence, MO, USA).

Consent All patients provided informed written consent prior to being interviewed.

Author contributions Jianming He, Ashley Duenas, Hannah Collacott, Annette Lam, Katherine Gries, Dietrich Potthoff, Nicola Trevor, and Tommi Tervonen were involved in the study conception and design. Ashley Duenas, Hannah Collacott, and Tommi Tervonen performed the formal data analysis. All authors were involved in the interpretation of data, contributed to the drafting of the manuscript, and approved the final version.

Open Access This article is licensed under a Creative Commons Attribution-NonCommercial 4.0 International License, which permits any non-commercial use, sharing, adaptation, distribution and reproduction in any medium or format, as long as you give appropriate credit to the original author(s) and the source, provide a link to the Creative Commons licence, and indicate if changes were made. The images or other third party material in this article are included in the article's Creative Commons licence, unless indicated otherwise in a credit line to the material. If material is not included in the article's Creative Commons licence and your intended use is not permitted by statutory regulation or exceeds the permitted use, you will need to obtain permission directly from the copyright holder. To view a copy of this licence, visit http://creativecommons.org/licenses/by-nc/4.0/.

\section{References}

1. Ramsenthaler C, Kane P, Gao W, Siegert RJ, Edmonds PM, Schey $\mathrm{SA}$, et al. Prevalence of symptoms in patients with multiple myeloma: a systematic review and meta-analysis. Eur J Haematol. 2016;97(5):416-29. https://doi.org/10.1111/ejh.12790.

2. Baz R, Lin HM, Hui AM, Harvey RD, Colson K, Gallop K, et al. Development of a conceptual model to illustrate the impact of multiple myeloma and its treatment on health-related quality of life. Support Care Cancer. 2015;23(9):2789-97. https://doi. org/10.1007/s00520-015-2644-6.

3. Parsons JA, Greenspan NR, Baker NA, McKillop C, Hicks LK, Chan O. Treatment preferences of patients with relapsed and refractory multiple myeloma: a qualitative study. BMC Cancer. 2019;19(1):264. https://doi.org/10.1186/s12885-019-5467-x.

4. Seitzler S, Finley-Oliver E, Simonelli C, Baz R. Quality of life in multiple myeloma: considerations and recommendations. Expert Rev Hematol. 2019;12(6):419-24. https://doi.org/10.1080/17474 086.2019.1613886.

5. Osborne TR, Ramsenthaler C, Siegert RJ, Edmonds PM, Schey SA, Higginson IJ. What issues matter most to people with multiple myeloma and how well are we measuring them? A systematic review of quality of life tools. Eur J Haematol. 2012;89(6):43757. https://doi.org/10.1111/ejh.12012.

6. Robinson D Jr, Esseltine DL, Regnault A, Meunier J, Liu K, van de Velde $\mathrm{H}$. The influence of baseline characteristics and disease stage on health-related quality of life in multiple myeloma: findings from six randomized controlled trials. Br J Haematol. 2016;174(3):368-81. https://doi.org/10.1111/bjh.14058.

7. Richardson PG, Schlossman RL, Roy AN, Panneerselvam A, Acharyya S, Sopala M, et al. Patient-reported outcomes of multiple myeloma patients treated with panobinostat after $\geq 2$ lines of therapy based on the international phase 3, randomized, double-blind, placebo-controlled PANORAMA-1 trial. Br J Haematol. 2018;181(5):628-36. https://doi.org/10.1111/bjh.15248.

8. Weisel K, Dimopoulos M, Moreau P, Yagci M, Larocca A, Kanate AS, et al. Health-related quality-of-life results from the phase 3 OPTIMISMM study: pomalidomide, bortezomib, and low-dose dexamethasone versus bortezomib and low-dose dexamethasone in relapsed or refractory multiple myeloma. Leuk Lymphoma. 2020. https://doi.org/10.1080/10428194.2020.1747066

9. Nielsen LK, Stege C, Lissenberg-Witte B, van der Holt B, Mellqvist UH, Salomo M, et al. Health-related quality of life in transplant ineligible newly diagnosed multiple myeloma patients treated with either thalidomide or lenalidomide-based regimen until progression: a prospective, open-label, multicenter, randomized, phase 3 study. Haematologica. 2020;105(6):1650-9. https ://doi.org/10.3324/haematol.2019.222299.

10. Postmus D, Richard S, Bere N, van Valkenhoef G, Galinsky J, Low E, et al. Individual trade-offs between possible benefits and risks of cancer treatments: results from a stated preference study with patients with multiple myeloma. Oncologist. 2018;23(1):44 51. https://doi.org/10.1634/theoncologist.2017-0257.

11. Mols F, Oerlemans S, Vos AH, Koster A, Verelst S, Sonneveld P, et al. Health-related quality of life and disease-specific complaints among multiple myeloma patients up to $10 \mathrm{yr}$ after diagnosis: results from a population-based study using the PROFILES registry. Eur J Haematol. 2012;89(4):311-9. https://doi.org/10.111 $1 / \mathrm{j} .1600-0609.2012 .01831 . x$.

12. Gulbrandsen N, Hjermstad MJ, Wisloff F, Nordic Myeloma Study Group. Interpretation of quality of life scores in multiple myeloma by comparison with a reference population and assessment of the clinical importance of score differences. Eur J Haematol. 2004;72(3):172-80. https://doi.org/10.104 6/j.0902-4441.2003.00195.x.

13. Sherman AC, Simonton S, Latif U, Plante TG, Anaissie EJ. Changes in quality-of-life and psychosocial adjustment among multiple myeloma patients treated with high-dose melphalan and autologous stem cell transplantation. Biol Blood Marrow Transpl. 2009;15(1):12-20. https://doi.org/10.1016/j.bbmt.2008.09.023.

14. Rifkin RM, Bell JA, DasMahapatra P, Hoole M, Lowe M, Curran $\mathrm{C}$, et al. Treatment satisfaction and burden of illness in patients with newly diagnosed multiple myeloma. Pharmacoecon Open. 2020;4(3):473-83. https://doi.org/10.1007/s41669-019-00184-9.

15. Postmus D, Mavris M, Hillege HL, Salmonson T, Ryll B, Plate A, et al. Incorporating patient preferences into drug development and regulatory decision making: results from a quantitative pilot study with cancer patients, carers, and regulators. Clin Pharmacol Ther. 2016;99(5):548-54. https://doi.org/10.1002/cpt.332.

16. de Bekker-Grob EW, Berlin C, Levitan B, Raza K, Christoforidi $\mathrm{K}$, Cleemput I, et al. Giving patients' preferences a voice in medical treatment life cycle: the PREFER public-private project. Patient. 2017;10(3):263-6. https://doi.org/10.1007/s4027 1-017-0222-3.

17. Johnson FR, Zhou M. Patient preferences in regulatory benefit-risk assessments: a US perspective. Value Health. 2016;19(6):741-5. https://doi.org/10.1016/j.jval.2016.04.008.

18. Muhlbacher AC, Bridges JF, Bethge S, Dintsios CM, Schwalm A, Gerber-Grote A, et al. Preferences for antiviral therapy of chronic hepatitis C: a discrete choice experiment. Eur J Health Econ. 2017;18(2):155-65. https://doi.org/10.1007/s10198-016-0763-8.

19. Francis JJ, Johnston M, Robertson C, Glidewell L, Entwistle V, Eccles MP, et al. What is an adequate sample size? Operationalising data saturation for theory-based interview studies. Psychol Health. 2010;25(10):1229-45. https://doi.org/10.1080/08870 440903194015.

20. Hollin IL, Craig BM, Coast J, Beusterien K, Vass C, DiSantostefano R, et al. Reporting formative qualitative research to support the development of quantitative preference study protocols and 
corresponding survey instruments: guidelines for authors and reviewers. Patient. 2020;13(1):121-36. https://doi.org/10.1007/ s40271-019-00401-x.

21. Friese S. ATLAS.ti 7 User Guide and Reference.

22. Friese S, Soratto J, D. P. Carrying out a computer-aided thematic content analysis with ATLAS.ti MMG Working Paper.

23. Turner-Bowker DM, Lamoureux RE, Stokes J, Litcher-Kelly L, Galipeau N, Yaworsky A, et al. Informing a priori sample size estimation in qualitative concept elicitation interview studies for clinical outcome assessment instrument development. Value Health. 2018;21(7):839-42. https://doi.org/10.1016/j. jval.2017.11.014.

24. von Elm E, Altman DG, Egger M, Pocock SJ, Gotzsche PC, Vandenbroucke JP, et al. Strengthening the Reporting of Observational Studies in Epidemiology (STROBE) statement: guidelines for reporting observational studies. BMJ. 2007;335(7624):806-8. https://doi.org/10.1136/bmj.39335.541782.AD.
25. Tong A, Sainsbury P, Craig J. Consolidated criteria for reporting qualitative research (COREQ): a 32-item checklist for interviews and focus groups. Int J Qual Health Care. 2007;19(6):349-57. https://doi.org/10.1093/intqhe/mzm042.

26. Wilke T, Mueller S, Bauer S, Pitura S, Probst L, Ratsch BA, et al. Treatment of relapsed refractory multiple myeloma: which new PI-based combination treatments do patients prefer? Patient Prefer Adherence. 2018;12:2387-96. https://doi.org/10.2147/PPA.S1831 87.

27. Warde F, Papadakos J, Papadakos T, Rodin D, Salhia M, Giuliani M. Plain language communication as a priority competency for medical professionals in a globalized world. Can Med Educ J. 2018;9(2):e52-9.

28. Smith MY, Wallace LS. Reducing drug self-injection errors: a randomized trial comparing a "standard" versus "plain language" version of Patient Instructions for Use. Res Social Adm Pharm. 2013;9(5):621-5. https://doi.org/10.1016/j.sapharm.2012.10.007. 